\title{
A new grid-cell-based method for error evaluation of vector-to-raster conversion
}

\author{
Shunbao Liao • Yan Bai
}

Received: 28 May 2009 / Accepted: 12 October 2009 / Published online: 28 October 2009

(C) The Author(s) 2009. This article is published with open access at Springerlink.com

\begin{abstract}
Error evaluation of rasterization of vector data is one of the most important research topics in the field of geographical information systems. Current methods for evaluating rasterization errors are far from perfect and need further improvement. The objective of this study is to introduce a new error evaluation method that is based on grid cells (EEM-BGC). The EEM-BGC follows four steps. First, the area of each land category inside a square is represented in a vector format. The size and location of the square are exactly the same as those of a grid cell that is to be generated by rasterization. Second, the area is treated as the attribute of the grid cell. Vector data are rasterized into $n$ grids, where $n$ is the number of land categories. Then, the relative area error resulting from rasterization for each land category in the grid cell is calculated in raster format. Lastly, the average of the relative area error for all land categories in the grid cell is computed with the area of a land category as weight. As a case study, the EEM-BGC is applied for evaluating the rasterization error of the land cover data of Beijing at a scale of 1 to 250,000 . It is found that the error derived from a conventional method (denoted as $y$ ) is significantly underestimated in comparison with that derived from the new method (denoted as $x$ ), with $y=0.0014 x^{2.6667}$.
\end{abstract}

\section{S. Liao $(\bowtie) \cdot$ Y. Bai}

LREIS, Institute of Geographic Sciences and Natural

Resources Research, Chinese Academy of Sciences,

Beijing 100101, People's Republic of China

e-mail: liaosb@igsnrr.ac.cn

\section{Y. Bai}

Graduate University of Chinese Academy of Sciences,

Beijing 100049, People's Republic of China
The EEM-BGC is effective in capturing not only the spatial distribution of rasterization errors at the gridcell level but also the numerical distribution range of the errors. The EEM-BGC is more objective and accurate than any conventional method that is used for evaluating rasterization errors.

Keywords Rasterization • Error evaluation • Grid cell

\section{Introduction}

Vector and raster are the two basic formats of geospatial data used in geographical information systems (GIS) [1]. Raster data are more suitable for spatial modeling and spatial analysis than vector data $[2,3]$. As the technologies of geospatial information collection including remote sensing (RS) and global positioning system (GPS) advance forward raster data have become more and more popular than vector data. Raster is now the most dominant format of data sources. The researches related to raster data processing, storage, analysis, and application have once again drawn a lot of attention in the GIS field [4, 5]. Data products in raster format are increasing rapidly. Therefore, rasterization of vector data is beneficial for performing comprehensive analysis of GIS data in different formats.

One advantage of rasterization is its scaling capability. For example, to produce a small-scale map from a large-scale map in the same zone one can choose either cartographic generalization or rasterization. Cartographic generalization usually needs a long mapping period and requires expert knowledge. On the contrary, rasterization costs much less time for mapping and does 
not require any expert knowledge for accomplishing the task. Cheng et al. [6] carried out data scaling by rasterizing vector data at a scale of 1 to 1,000 to produce a set of databases at the resolutions of $5,10,15,20$, and $30 \mathrm{~m}$, respectively. Liao and Sun [7] and Liao et al. [8] did scaling by rasterizing census population data from administrative divisions to regular grids.

Another advantage of rasterization lies in that rasterized data products are convenient for data sharing. In most cases vector data of large scales are subject to information security and intellectual property protection. These data become publicly accessible as their spatial resolution decreases after rasterization. We introduce below rasterization errors and describe the conventional method used for evaluating rasterization errors.

\subsection{Rasterization errors}

As does cartographic generalization rasterization inevitably generates errors. It is a conversion process accompanied with information loss. Errors always exist no matter how precise the conversion process is. For the rasterization of polygonal features, errors are found in area, perimeter, shape, structure, position, and attributes [9]. These errors vary as data source, rasterization algorithm, and data structure change [10]. Given the same input, the outcome may still be different because a variety of rasterization algorithms are used [11]. Therefore, error evaluation is an important component of rasterization.

Shortridge [12] showed that rasterization errors are sensitive to cell size, polygonal shape, and structure. Burrough and McDonnell [13] discussed the sources of rasterization errors in detail. However, neither of them investigated error evaluation and error reduction. Frolov and Maling [14] presented a probability statistical model for the error analysis of vector-to-raster conversion of polygons. Bregt et al. [15] proposed an error analysis method that combines dual conversion and boundary index. Zhou et al. [16] presented an equal area conversion model for rasterization, which minimizes the error in area at the cost of losing boundary features. In view of the limitations of current rasterization methods, $\mathrm{Wu}$ et al. [20] proposed a winding number algorithm based on the rotation angle theory in computational geometry. This method is simple but is highly effective and easy to use. Wang et al. [17] summarized current existing algorithms and proposed an optimization algorithm which is aimed to minimize the error in area after vector-to-raster conversion.

A good example that can be used to demonstrate the cause of errors for polygonal rasterization is the representation of mixed pixels in remotely sensed data. A single pixel in remote sensing images with middle or low resolutions often consists of more than one land category. Its spectral value is the sum of spectrum of all land categories within the pixel. The degree of complexity increases with the number of land categories residing in the pixel. Usually there are more mixed pixels along the boundary of a land cover than inside [18]. The existence of mixed pixels is one of the major causes of low interpretation accuracy in remote-sensing images. In modern remote-sensing technology and applications, new techniques such as linear, probabilistic, and geometric-optical models have been developed to decompose mixed pixels [19]. It is necessary to point out that although rasterization errors and mixed pixels share certain commonality, they are intrinsically different. The progress made on the representation and evaluation of rasterization error also falls behind the development of mixed-pixel decomposition. More research is needed to improve the accuracy of rasterization-error evaluation.

\subsection{Evaluation of rasterization errors}

In most cases vector data can be rasterized following two theorems, the greatest area theorem and the central point theorem. The conventional approaches for the evaluation of rasterization errors include: (1) compute the area of each land category $\left(A_{0}^{i}\right)$ in vector format before rasterization and treat it as a reference for this land category; (2) compute the area of each land category $\left(A^{i}\right)$ in grid format after rasterization; and (3) calculate the error of rasterization for each land category using the following equation,

$E^{i}=\left(A^{i}-A_{0}^{i}\right) / A_{0}^{i}$

where $i=1,2, \ldots, n$,is the index of land category, $E^{i}$ represents the relative error of rasterization for each land category $i$. Positive (negative) $E^{i}$ indicates that the grid area after rasterization is larger (smaller) than the vector area before rasterization for a certain land category. The larger the absolute value is, the bigger the error is.

Using the aforementioned method Yang and Zhang [21] rasterized the land-use data in Chongqing at a scale of 1 to 100,000 and investigated the degree of precision losses for all land categories with respect to different grid-cell sizes. They found that the relationship among precision loss, the average size of polygonal patch, and grid cell size can be well represented with a model. Based on the greatest area theorem, Liu et al. [22] rasterized the land-use and land cover data of China at a scale of 1 to 100,000 and produced a grid database at a 
resolution of $1 \times 1 \mathrm{~km}$. They analyzed the rasterization error and concluded that the relative error between the actual area in vector format and the area resulting from rasterization is in proportion to the complexity of the land use and land cover within a given region. The more complex the region is the larger the error becomes.

A major problem of the conventional method used for evaluating rasterization errors is that the evaluation is based on the entire region rather than each grid cell. It focuses on the total error in the entire study region and ignores the spatial distribution of errors, which often increase in one cell but decrease in another. As a consequence, for most cases rasterization errors are greatly underestimated [23]. Total error cannot reflect the irregularity of errors in the entire region. Ignoring local errors can cause the loss of important information and even lead to wrong conclusions [24].

To improve the accuracy of error analysis in vectorto-raster conversion, Chen et al. [23] proposed to make use of structural raster data. This method does not have the limitation of the conventional one which has only one error estimate in the entire region and thus is more objective and accurate. It is convenient for generating and visualizing error maps. The study greatly improved the evaluation of rasterization errors. Nevertheless, there are still two questions remained to be answered.

First, the study introduced a new index called Precision of Category, which is the inverse of the number of categories inside a grid cell and is independent of the categories' area. Its significance and purpose were not well defined. For example, in Fig. 1 there are four land categories: class 1 , class 2 , class 3 , and class 4 , in each of the two grids: grid 1 and grid 2. The percent areas occupied by each class in the two cells are different. According to the definition of Chen et al. [23], the precisions of category for grid 1 and grid 2 are the same, i.e., 1/4. However, the actual rasterization precision losses between the two grids are evidently different.

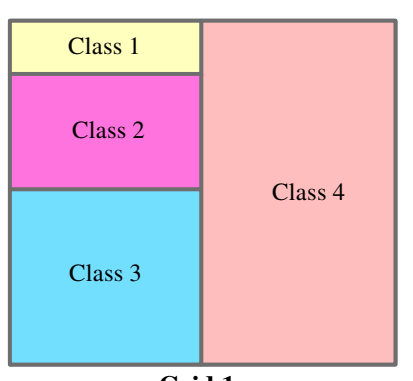

Grid 1

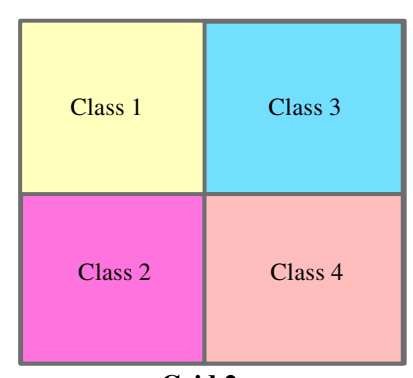

Grid 2
Fig. 1 An illustration to show that two grid cells have the same number of classes but the areas occupied by each class are different between the two cells
Second, the study did not explain how to compute the overall rasterization error in each grid cell even though it did calculate and analyze the error for each land cover type within the cell. This makes the comparison of errors from different rasterization methods difficult. For different data sources and/or different cell sizes, the order of magnitude of errors for a certain land type varies in different rasterization schemes. Taking a vector map which is composed of three land classes as an example, the map is rasterized with two different schemes. Assuming that the errors of the three land classes resulting from the first scheme are $18 \%, 15 \%$, and $21 \%$, respectively, and the corresponding errors from the second scheme are $17 \%, 18 \%$, and $19 \%$. It is impossible to tell which scheme has a higher overall precision. Therefore, it is imperative to construct a general index for the evaluation of rasterization errors. This index shall cover all land categories within a grid cell.

In this study, we introduce a new error evaluation method that is based on grid cells, namely, the EEMBGC method. In Section 2 we introduce this new method. The EEM-BGC is then applied in Section 3 for evaluating the rasterization error of the land cover data of Beijing at a scale of 1 to 250,000. Section 4 summarizes this study.

\section{Error evaluation method based on grid cells}

Suppose that vector data are composed of $n$ polygons of land categories inside a given grid cell. They are labeled as $P_{1}, P_{2}, \ldots$, and $P_{n}$, respectively. Their areas are $A_{1}$, $A_{2}, \ldots, A_{n}$, and the total area is $A$, that is,

$A=\sum_{i=1}^{n} A_{i}$

It is further assumed that polygon $P_{n}$ has the largest area among all the polygons (see Fig. 2). Based on the largest area theorem, category attribute of the grid cell

Fig. 2 A grid cell with $n$ land categories

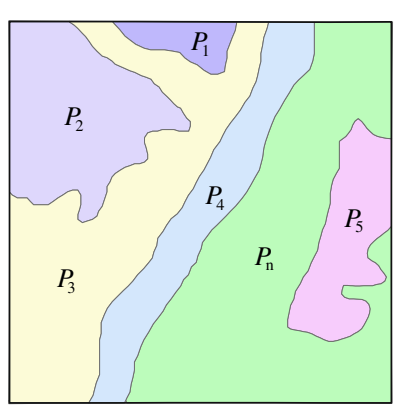


is replaced by the attribute of polygon $P_{n}$ after it is rasterized. This results in area gain or loss for each polygon of the land category inside the grid cell. The details are given below:

1. The areas of polygons $P_{1}, P_{2}, \ldots$, and $P_{n-1}$ are $A_{1}, A_{2}, \ldots, A_{n-1}$ before rasterization. They are all reduced to zero after rasterization. The absolute errors are $A_{1}, A_{2}, \ldots, A_{n-1}$, respectively, and the relative errors are all one by Eq. 1 .

2. The area of polygon $P_{n}$ is $A_{n}$ before rasterization and $A$ after rasterization. The absolute error is $\left(A-A_{n}\right)$ and relative error is $\left(A-A_{n}\right) / A_{n}$.

The relative error is more scientifically meaningful and reasonable than the absolute error. Polygons of different patch sizes contribute differently to the general (total) error even if they have the same relative errors. Similarly, given the same relative error the larger area a polygon has the larger a contribution to the general error of a grid cell it makes. Therefore, it is justifiable to use polygon area as weight to calculate the general relative error of a grid cell, that is,

$\mathrm{RE}=\sum_{i=1}^{n-1}\left[1 \times\left(A_{i} / A\right)\right]+\left[\left(A-A_{n}\right) / A_{n}\right] \times\left(A_{n} / A\right)$

From Eq. 2 we have $\sum_{i=1}^{n-1} A_{i}=A-A_{n}$. Therefore, Eq. 3 can be rewritten as

$\mathrm{RE}=2\left(A-A_{n}\right) / A$

It is easy to show that $\mathrm{RE}$ is in the range of $[0,2]$. To make it more consistent with the commonly used relative error that is in the range of $[0,1]$, we divide $\mathrm{RE}$ by a factor of 2 , that is,

$\mathrm{RE}=\left(A-A_{n}\right) / A$

Equation 5 shows that: (1) the closer the area of a category that has the largest size is to the total area of the grid cell, the less the relative error of the grid cell is; (2) the more number of categories a grid cell contains, the larger the relative error of rasterization is. In general, multiple categories lead to area reductions of all polygons, including the polygon that has the largest size.

As an example, we use Eq. 5 to calculate the relative errors of the grid cells shown in Fig. 1. Suppose the area of each grid cell is $a$, then we have $\mathrm{RE}=(a-0.5 a) / a=$ 0.5 for grid 1 , and $\mathrm{RE}=(a-0.25 a) / a=0.75$ for grid 2 .
One can see that the general relative errors between the two grids are different.

\section{A case study}

In this section we apply the EEM-BGC method introduced in Section 2 to evaluate the rasterization errors of the land cover data of Beijing at different grid cell sizes. Results are compared with those made with convectional methods to assess the accuracy of the EEMBGC method.

\subsection{Data sources}

This study uses the land cover database of Beijing at a scale of 1 to 250,000 , which is a part of the land cover database of China at the same scale. This database categorizes the land covers of China into six level-1 classes and 25 level-2 classes.

There are five level- 1 classes and 15 level- 2 classes of land cover in Beijing. They are evergreen coniferous forest, deciduous needle-leaf forest, deciduous broadleaved forest, mixed wood, shrub, meadow grassland, typical grassland, shrub grassland, paddy field, irrigated land, dry land, urban construction land, rural settlement, inland water, and river or lake beach. The area of each land cover category and its proportion to the total area are given in Table 1. Their spatial distributions are presented in Fig. 3.

Table 1 Land cover categories and the corresponding areas in Beijing

\begin{tabular}{llrr}
\hline $\begin{array}{l}\text { Code } \\
\text { number }\end{array}$ & Land cover category & $\begin{array}{l}\text { Area } \\
\text { (ha) }\end{array}$ & $\begin{array}{c}\text { Percent } \\
\text { area (\%) }\end{array}$ \\
\hline 11 & Evergreen coniferous forest & 423 & 0.03 \\
13 & Deciduous needle-leaf forest & 59,257 & 3.62 \\
14 & Deciduous broad-leaved forest & 342,859 & 20.92 \\
15 & Mixed wood & 160,447 & 9.79 \\
16 & Shrub & 190,730 & 11.64 \\
21 & Meadow grassland & 21,895 & 1.34 \\
22 & Typical grassland & 9,502 & 0.58 \\
26 & Shrub grassland & 92,448 & 5.64 \\
31 & Paddy field & 18,959 & 1.16 \\
32 & Irrigated land & 400,022 & 24.41 \\
33 & Dry land & 56,066 & 3.42 \\
41 & Urban construction land & 146,926 & 8.97 \\
42 & Rural settlement & 93,345 & 5.70 \\
53 & Inland water and & 24,520 & 1.50 \\
54 & River and lake beach & 21,179 & 1.29 \\
Total & & $1,638,577$ & 100.00 \\
\hline
\end{tabular}


Fig. 3 Land cover map of Beijing at a scale of 1 to 250,000

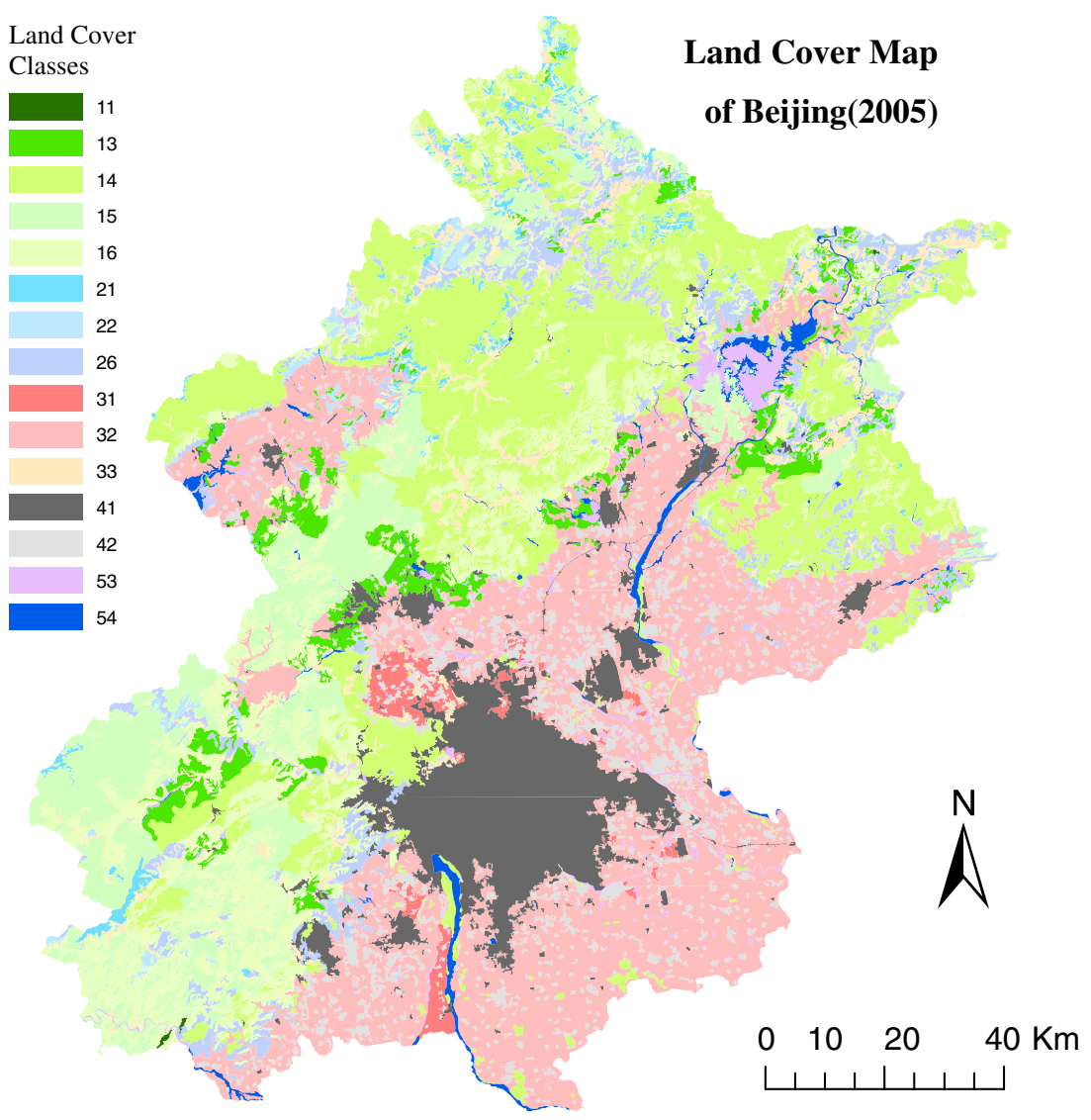

\subsection{Rasterization method}

The ESRI ArcGIS software is employed to compute the percent area of each land cover category within a square relative to the total area of the square, which has a size of $100 \times 100 \mathrm{~m}$ and is in vector-data format. The percentage of area is then used as a key attribute value of the vector square to generate rasterized grid data at a resolution of $100 \times 100 \mathrm{~m}$.

For example, in Fig. 4 there are $n$ polygons representing $n$ different types of land cover. The area percentages of polygons $p_{1}, p_{2}, \ldots, p_{n}$ to the entire square can be readily computed and converted from float numbers to integers by using the ArcGIS software. The resulting percentages are $7 \%, 27 \%, \ldots$, and $40 \%$. After rasterization, $n$ grids are generated and their cell values are $7,27, \ldots$, and 40 , respectively.

Special attention should be paid to the cases for which different polygons inside a square represent the same category of land cover. In such a case, all area percentages of the same category should be assembled together and treated as the grid-cell value of this category.

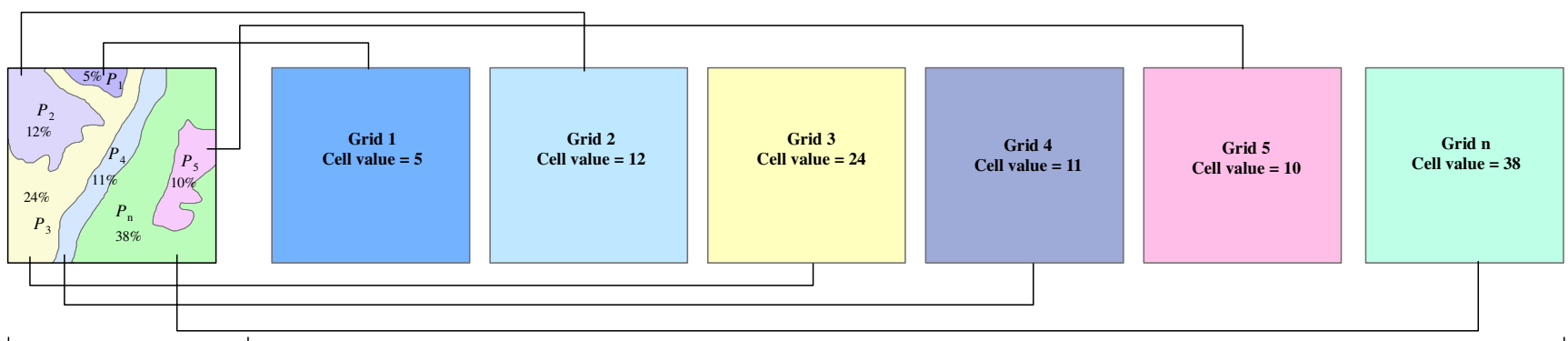

Vector Data

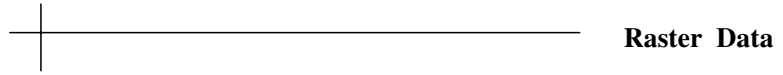

Fig. 4 Schematic diagram showing that area information is kept after rasterization 
Zero grid-cell values are assigned to those land cover categories that do not exist in a square.

The classification system of Beijing's land cover consists of 15 level-2 classes. Therefore, 15 grids are generated (see Fig. 5). The cell value of each grid represents the area percentage of a category in the $100 \times$ $100 \mathrm{~m}$ grid cells. In Comparison with conventional rasterization methods, the EEM-BGC method conserves the area information of each land cover category in all grid cells. UNION and POLYGRID are the two key operations in this step. The UNION computes the geometric intersection of two polygon coverages, the coverage of land cover in Beijing and the fishnet coverage of grid cell in vector format. All polygons from the two coverages will be split at their intersections and preserved in the output coverage. The POLYGRID creates 15 grids from the vector-format polygonal coverage.
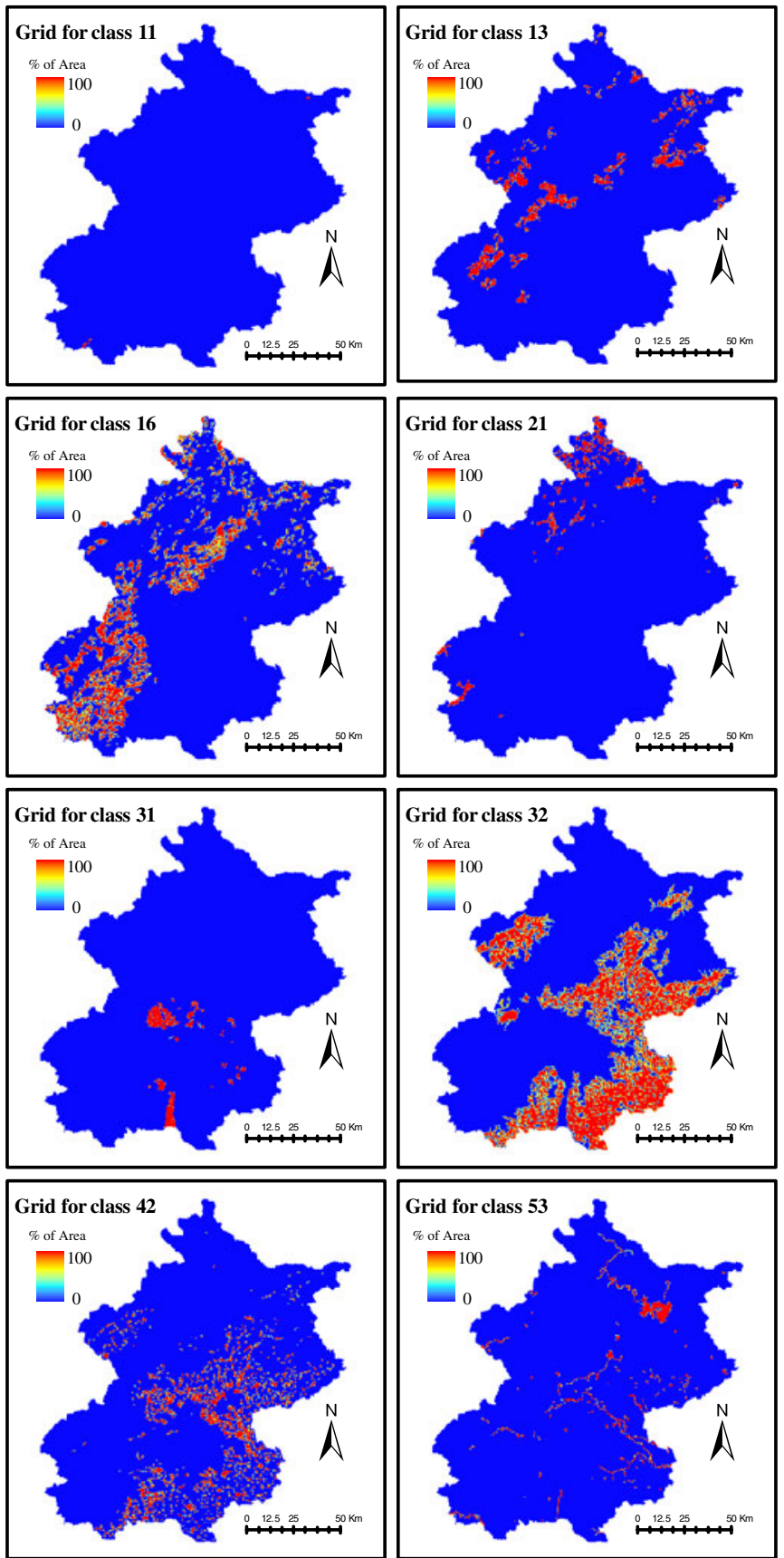
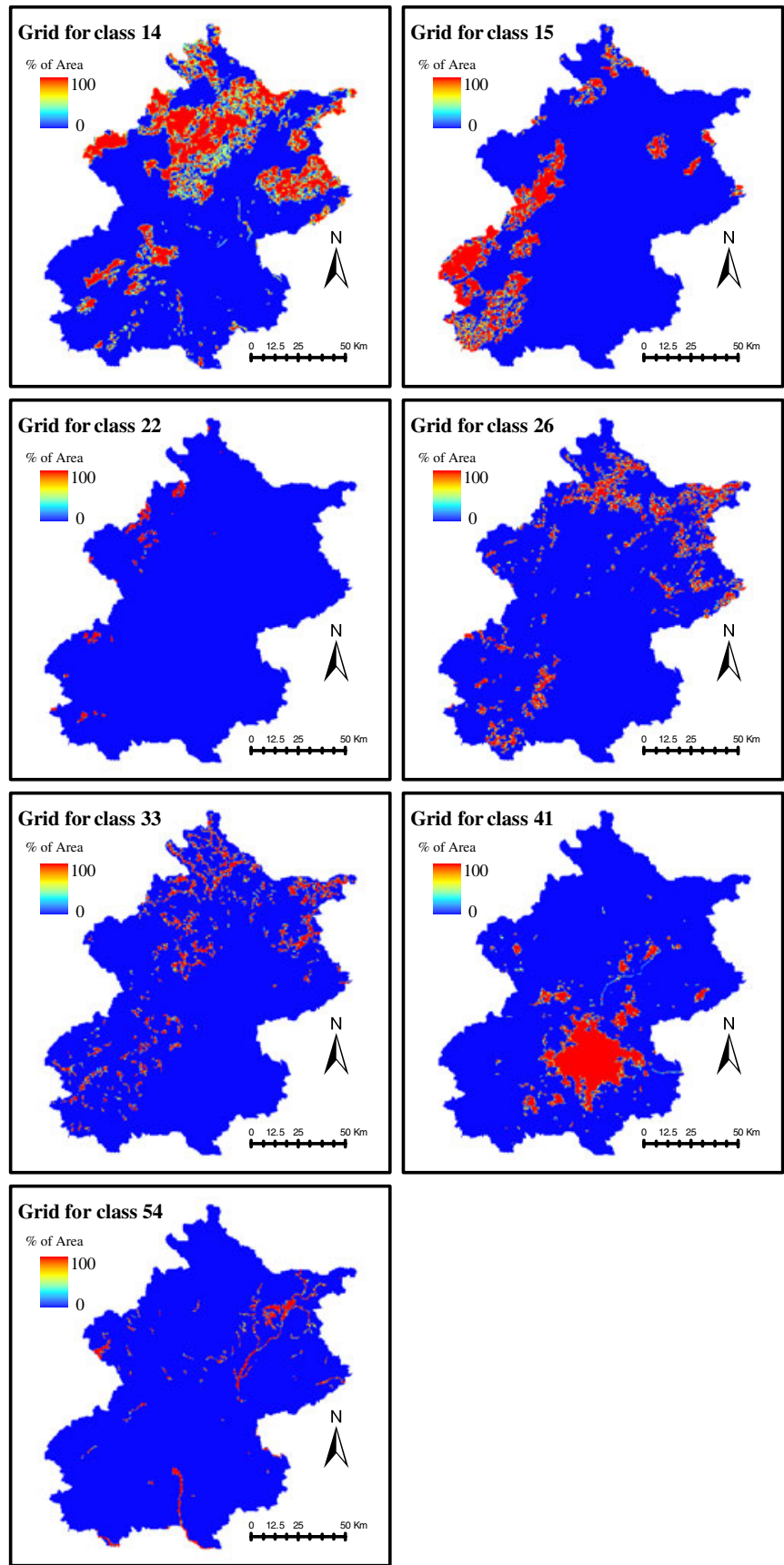

Fig. 5 Rasterization result of land cover map of Beijing without any loss of area information 


\subsection{Error calculations}

Error calculations are accomplished through the following three steps.

Step 1: Find the area percentage of the land cover category which has the largest area among all polygons within each grid cell, that is, the $A_{n}$ in Eq. 5. The function max in the ArcGIS system provides such a tool for this calculation.

- Max_Value_Grid $=\max (\operatorname{grid} 1, \operatorname{grid} 2, \ldots$, grid15)

The result is shown in Fig. 6.

Step 2: Calculate the general relative error on each grid cell using Eq. 5.

- Cell_Avg_Err $=(100-$ Max_Value Grid)/100.

Step 3: Calculate average relative error for the entire region in question, i.e., Beijing, based on the result from step 2.

Region_Avg_Err $=$ zonalmean $($ Region_ Boundary_Grid, Cell_Avg_Err).
Where Cell_Avg_Err is a value grid representing general relative error of each grid cell in the study area (Beijing). Region_Boundary_Grid is a zone grid of the study area with the same resolution as Cell_Avg_Err. The function zonalmean records in each output cell the mean of the values of all cells in the value grid (Cell_Avg_Err) that belongs to the same zone as the output cell. Zones are identified by the values of the cells in Region_Boundary_Grid.

The above three steps are repeated to obtain general relative errors for each grid cell and average relative errors for the entire study area at different resolutions, i.e., $200 \times 200 \mathrm{~m}, 500 \times 500 \mathrm{~m}, 1 \times 1 \mathrm{~km}, 2 \times 2 \mathrm{~km}, 5 \times$ $5 \mathrm{~km}$, and $10 \times 10 \mathrm{~km}$, respectively.

\subsection{Analysis of results}

\section{Spatial distribution of errors}

Presented in Fig. 7 is the spatial distribution map of the relative errors at grid cell level. It can be seen that (1) the errors are mostly found along the boundary lines of the vector polygons of different land cover categories. The smaller a grid cell is, the more the errors occur along the boundary lines; (2) the general error becomes larger as the grid cell gets larger; (3) the errors in the north and southwest regions of Beijing is larger than
Fig. 6 A grid that shows the largest percentage of area

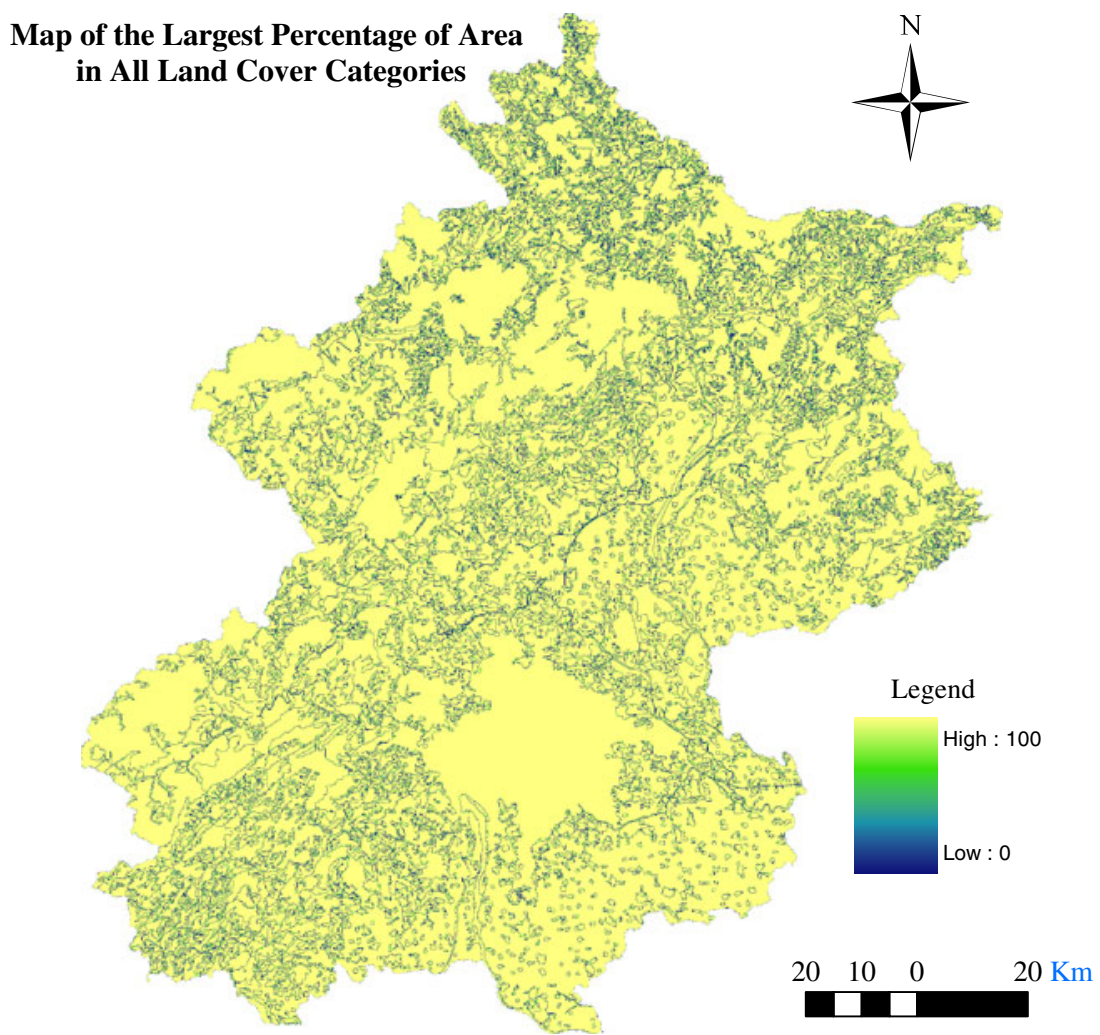


Fig. 7 Spatial distribution of errors at different grid resolutions
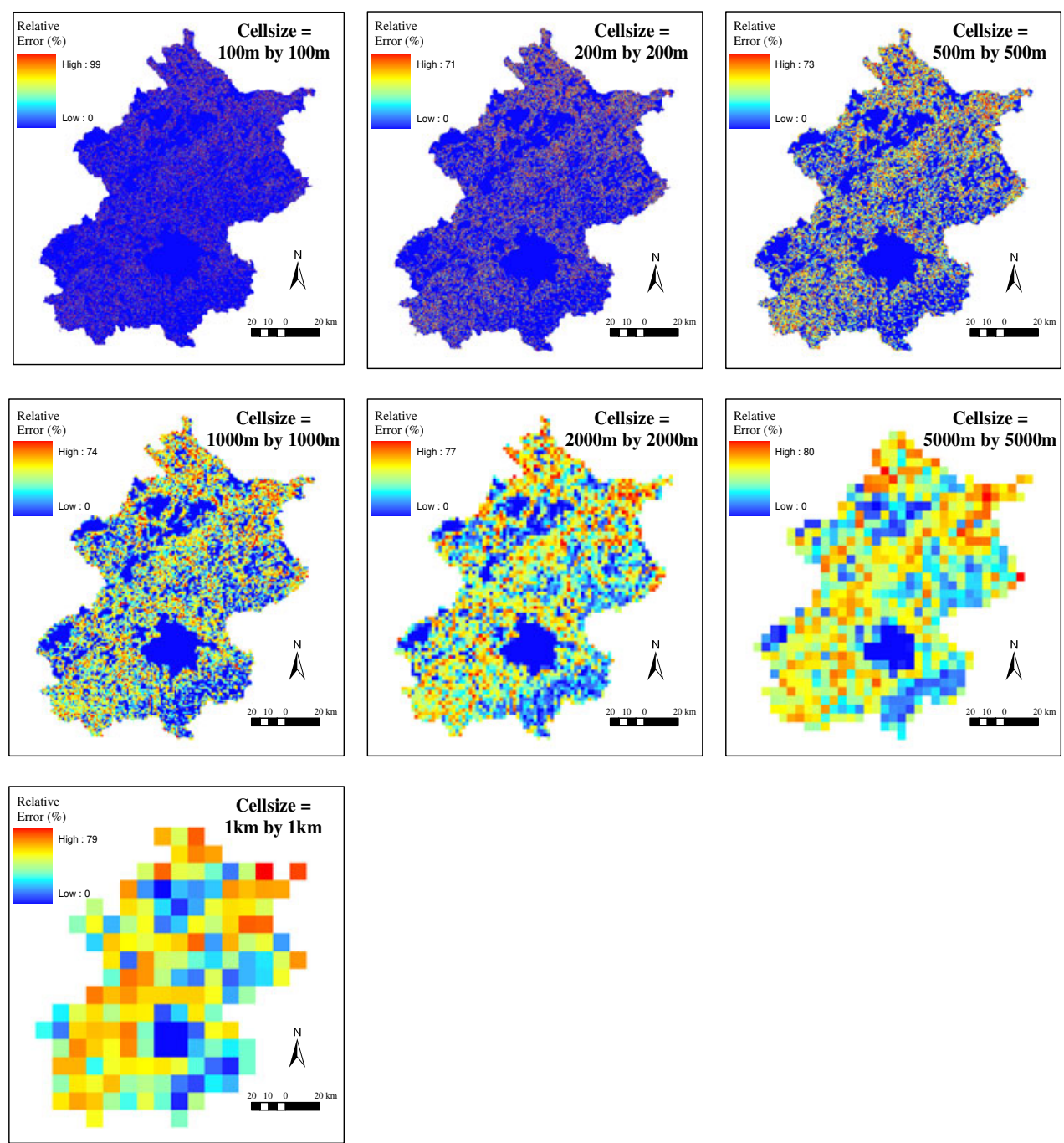

3. Comparison with the conventional method of error evaluation

district has the lowest zero error.

\section{Numerical distribution of errors}

Shown in Fig. 8 are the numerical distribution charts of relative errors for all rasterization grid cell sizes. One can see that as the grid cell size increases the percentage of grid cells of lower errors decreases and the percentage of grid cells of higher errors increases. For instance, for a grid cell size of $100 \times 100 \mathrm{~m}$, about $80 \%$ of the grid cells have relative errors close to zero, and only $1 \%$ of the grid cells have relative errors larger than $50 \%$. As the grid cell size increases to $1 \mathrm{~km}$, about $21 \%$ of the grid cells have relative errors close to zero, and $9 \%$ of the grid cells have relative errors exceeding $50 \%$. As the grid cell size increases to $10 \mathrm{~km}$, only $1 \%$ of the grid cells have relative errors close to zero, and about $50 \%$ of the grid cells have relative errors exceeding $50 \%$.
The conventional error evaluation method is based on the entire region rather than grid cells. To compare the conventional method with the EEM-BGC method introduced in this study, we computed the general relative error defined by the conventional evaluation method as the sum of the single relative error of each land cover category weighted by the area of the category,

$E=\sum_{i=1}^{15}\left(E_{i} \times f_{i}\right)$

Where $E$ is general relative error for the entire region, $E_{i}$ is general relative error for category $i, f_{i}$ is the percentage of area of category $i$ relative to total area. Table 2 compares the general relative errors obtained form the two methods. 
Fig. 8 Distributions of relative errors at different rasterization grid sizes
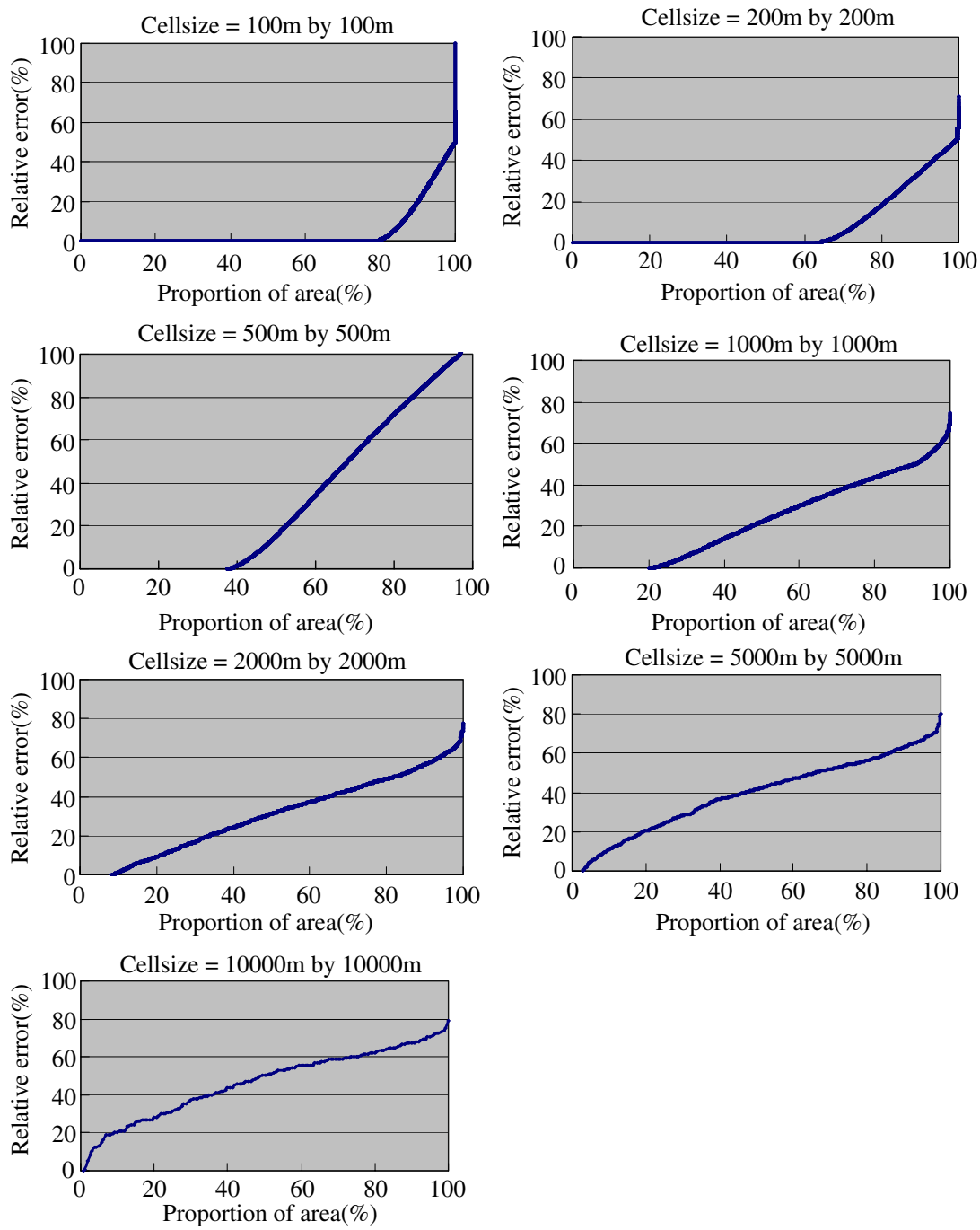

Table 2 shows that the general relative errors computed using the conventional error evaluation method are significantly smaller than that computed using the new method. The main reason for this discrepancy is that the conventional method treats the study region as a whole without considering the spatial distribution of errors. It ignores the fact that the area of one land

Table 2 Comparison of general relative errors between two error evaluation methods

\begin{tabular}{lcc}
\hline Grid cell size & \multicolumn{2}{c}{ General relative error $(\%)$} \\
\cline { 2 - 3 } & EEM-BGC & Conventional method \\
\hline $100 \times 100 \mathrm{~m}$ & 4.33 & 0.06 \\
$200 \times 200 \mathrm{~m}$ & 8.12 & 0.37 \\
$500 \times 500 \mathrm{~m}$ & 16.00 & 2.68 \\
$1,000 \times 1,000 \mathrm{~m}$ & 23.34 & 7.55 \\
$2,000 \times 2,000 \mathrm{~m}$ & 30.24 & 14.34 \\
$5,000 \times 5,000 \mathrm{~m}$ & 39.20 & 20.77 \\
$10,000 \times 10,000 \mathrm{~m}$ & 46.45 & 32.02 \\
\hline
\end{tabular}

cover type may decrease in one cell but increase in the other. Nevertheless, a close relation exists between the results from the two methods. A power fit of the general relative errors shown in Table 2 is presented in Fig. 9.

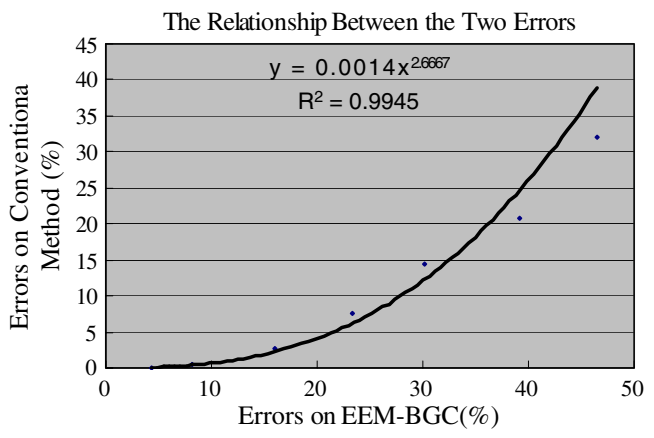

Fig. 9 Empirical fit between the errors computed using the conventional method for error evaluation and the EEM-BGC method 
The empirical relation included in Fig. 9 can be used to estimate the EEM-BGC based errors if the errors from the conventional error evaluation method are given, and vice versa.

\section{Summary and conclusion}

Vector and raster are two basic types of geospatial data. Conversion from vector to raster is one of the most important tools used by the community to process geospatial data or to produce data products. It is inevitable that rasterization generates errors. Rasterization is a conversion process accompanied by information losses. This study reviewed the progress on rasterization and shortcomings of current methods for evaluating rasterization errors and proposed a new error evaluation method based on grid cells, the EEM-BGC method. It was then used to rasterize the land cover data of Beijing at a scale of 1 to 250,000. From this study we conclude that

1. The conventional method for rasterization error evaluation, which is based on the level of the entire geographic area, cannot describe the spatial distribution and numerical distribution of errors.

2. In comparison with the conventional method for rasterization error evaluation, the EEM-BGC method is not only able to calculate more accurately the general errors of rasterization but is also able to describe more precisely the spatial and numerical distributions of the errors. It also makes it easy to compare the errors obtained from different rasterization methods. Furthermore, the EEMBGC method enhances the visualization capability. It is helpful for diagnosing the cause of errors and improving the precision of rasterization.

3. Error evaluation is an intrinsic part of the vectorto-raster conversion process. It is also an important component of the geo-science data quality control. The case study shows that rasterization error has a close relation with topology. In hill areas in the northern side of Beijing, rising and falling terrain leads to high complexity of land cover categories. Large errors may occur because polygonal patches of the land cover are tattered. Therefore, the precision of rasterization is determined by many factors such as data sources and rasterizing methods. Special attention should be paid to geographic background information including terrain and landforms for achieving accurate evaluation of rasterization errors.

Acknowledgements This research is supported by the State Key Laboratory of the Resources and Environment Information System (Grant O88RA100SA) and the Institute of Geographic Sciences and Natural Resources Research (Grant O66U0309SZ) of the Chinese Academy of Sciences. Their supports are gratefully acknowledged.

Open Access This article is distributed under the terms of the Creative Commons Attribution Noncommercial License which permits any noncommercial use, distribution, and reproduction in any medium, provided the original author(s) and source are credited.

\section{References}

1. Maguire, D.A., Goodchild, M.F., Rhind, D.W.: Geographical Information Systems: Principle, vol. 1, pp. 45-54. Longman Group UK Limited, Essex (1991)

2. Bates, P.D., Deroo, A.P.J.: A simple raster-based model for flood inundation simulation. J. Hydrol. 236, 54-77 (2000)

3. Demers, M.N.: GIS Modeling in Raster. Wiley, New York (2002)

4. Chen, S.P.: The time-and-space analysis of population statistics. China Population, Resources and Environment 12(4), 3-7 (2002)

5. Chen, S.P.: Grid mapping and grid computing. Science of Surveying and Mapping 27(4), 1-6 (2002)

6. Cheng, J.H., Wei, F.Y., Xue, H.Z.: Method for spatial data scale conversion. Geospatial Information 6(4), 13-15 (2008)

7. Liao, S.B., Sun, J.L.: GIS based spatialisation of population census data in Qinghai-Tibet Plateau. Acta Geogr. Sin. 58(1), 25-33 (2003)

8. Liao, Y.L., Wang, J.F., Meng, B., et al.: A method of spatialization of statistical population. Acta Geogr. Sin. 62(10), 1110-1119 (2007)

9. Carvers, S.J., Brunsdon, C.F.: Vector to raster error and feature complexity: an empirical study using simulated data. Int. J. Geogr. Inf. Syst. 8(3), 261-270 (1994)

10. Burrough, P.A.: Principles of Geographical Information Systems for Land Resources Assessment. Clarendon, Oxford (1986)

11. Van Der, K.: The vector to raster conversion: (mis) use in geographical information systems. Int. J. Geogr. Inf. Syst. 6(2), 159-170 (1992)

12. Shortridge, A.M.: Geometric variability of raster cell class assignment. Int. J. Geogr. Inf. Sci. 18(6), 539-558 (2004)

13. Burrough, P.A., Mcdonnell, R.A.: Principles of Geographic Information Systems, pp. 220-240. Oxford University Press, Oxford (1998)

14. Frolov, Y.S., Maling, D.H.: The accuracy of area measurement by point counting techniques. Cartogr. J. 6, 21-25(1969)

15. Bregt, A.K., Denneboom, J., Gesink, H.J., et al.: Determination of rastering error: a case study with the soil map of the Netherlands. Int. J. Geogr. Inf. Sci. 8, 261-367 (1991)

16. Zhou, C.H., Ou, Y., Yang, L., et al.: Equal-area-conversion from polygonal vector to raster. Sci. China, Ser. D Earth Sci. 36(s2), 157-163 (2006) 
17. Wang, X.L., Sun, Q.H., Jiang, C.S.: An optimization algorithm for transferring vector to raster data based on minimized area error. Acta Geodaetica et Cartographica Sinica 35(3), 273-277 (2006)

18. You, X.B., You, X.X., Xiang, Y.Y.: Mixed pixel and mixed pixel analysis. Journal of Beijing Forestry University 25(Spec. Iss.), 28-32 (2003)

19. Wei, J.J., Li, X.P., Zhao, D.B., et al.: Review on un-mixing mixed-pixel of remotely sensed data. Research of Soil and Water Conservation 13(5), 103-105 (2006)

20. Wu, G.C., Zuo, J.Z., Liu, Y. et al.: A high-effective algorithm for rasterization of vector data: winding number algorithm. Science of Surveying and Mapping 34(1), 50-52 (2009)
21. Yang, C.J., Zhang, Z.X.: Models of accuracy loss during rasterizing land use vector data with multiscale grid size. Geogr. Res. 20(4), 416-422 (2001)

22. Liu, M.L., Tang, X.M., Liu, J.Y., et al.: Research on scaling effect based on $1 \mathrm{~km}$ grid cell data. J Remote Sens. 5(3), 183$190(2001)$

23. Chen, J.J., Zhou, C.H., Cheng, W.M.: Area error analysis of vector to raster conversion of areal feature in GIS. Acta Geodaetica et Cartographica Sinica 36(3), 344-350 (2007)

24. Lunetta, R.S., Gongalton, R.G., Fenstermaker, L.K., et al.: Remote sensing and geographic information data integration: error sources and research issues. Photogramm. Eng. Remote Sensing 57(6), 677-687 (1991) 